\title{
Controle estratégico do carrapato Amblyomma cajennense em eqüinos
}

\author{
Strategic control of the tick Amblyomma cajennense on horses
}

\author{
Marcelo Bahia Labruna ${ }^{1}$ Romário Cerqueira Leite ${ }^{2}$ Alexandre Augusto de Oliveira Gobesso ${ }^{3}$ \\ Solange Maria Gennari ${ }^{4}$ Nobuko Kasai ${ }^{5}$
}

RESUMO

Avaliou-se quantitativamente as infestações por adultos de Amblyomma cajennense em eqüinos mantidos sob infestações naturais em uma pastagem (pastagem 1), antes e após o uso de tratamentos carrapaticidas. Os tratamentos foram aplicados nos eqüinos a cada sete dias durante os períodos de predomínio das larvas e ninfas do carrapato, no Estado de São Paulo (abril a outubro). Nos eqüinos mantidos em outra pastagem (pastagem 2), os tratamentos foram realizados, também a cada sete dias, mas de abril a julho, época de predomínio das larvas de A. cajennense. Todos os banhos carrapaticidas foram realizados com carrapaticida comercial à base do piretróide alfametrina A redução da carga de carrapatos adultos após os banhos carrapaticidas foi de 89,7 e 58,6\% nos eqüinos alocados nas pastagens 1 e 2, respectivamente. A redução das fêmeas adultas foi ainda mais expressiva (95,4 e 69,0\% para os eqüinos das pastagens 1 e 2, respectivamente). Os resultados comprovam a eficácia de uma proposta de controle estratégico de A. cajennense em eqüinos, baseada em banhos carrapaticidas intervalados de sete dias, nas épocas de predomínio dos estádios imaturos deste carrapato.

Palavras-chave: Amblyomma cajennense, controle estratégico, eqüinos.

\section{ABSTRACT}

Infestations by Amblyomma cajennense on horses grazing in a pasture (pasture 1) under natural conditions were evaluated quantitatively before and after acaricide treatments performed at seven day-intervals on the horses during the period of A. cajennense larvae and nymphs predomination on the horses (April to October). Horses grazing in another area (pasture 2) were subjected to acaricide treatments every seven days however, only during the larval predominating period (April to July). Acaricide treatments were performed using a commercial alfametrin (Pyrethroid) composition. The reduction of $\boldsymbol{A}$. cajennense adult tick loads on horses after the acaricide treatments was 89.7 and $58.6 \%$ for horses from pastures 1 and 2, respectively. The reduction values of adult female ticks were even greater (95.4 and 69.0\% for horses from pastures 1 and 2, respectively). The results comprove the efficacy of a strategic control protocol of $\boldsymbol{A}$. cajennense on horses which is based in acaricide treatments every seven days during the periods when infestations by $\boldsymbol{A}$. cajennense larvae and nymphs predominate on the horses.

Key words: Amblyomma cajennense, strategic control, equids.

\section{INTRODUÇÃO}

O carrapato Amblyomma cajennense, vulgarmente conhecido como carrapato-estrela, está amplamente difundido no território brasileiro. $\mathrm{Na}$ Região Sudeste, os eqüídeos se constituem nos principais hospedeiros desse carrapato. Entretanto, populações de $\boldsymbol{A}$. cajennense mantêm-se em áreas livres de eqüinos, parasitando várias espécies de animais silvestres (ARAGÃO, 1936). Dada a sua baixa especificidade, assume um importante papel na transmissão de patógenos entre os animais e o homem. De fato, A. cajennense tem sido reconhecido por seus ataques em massa, especialmente nas formas jovens, aos humanos quando em caminhada por matas e campos (ARAGÃO, 1911; 1936). A. cajennense completa apenas uma geração por ano na Região

${ }^{1}$ Médico Veterinário, Professor Doutor, Deparatamento de Medicina Veterinária Preventiva e Saúde Animal, Faculdade de Medicina Veterinária e Zootecnia (FMVZ), USP, Av. Prof. Orlando Marques de Paiva, 87, 05508-000, São Paulo, SP. E-mail: labruna@usp.br Autor para correspondência.

${ }^{2}$ Médico Veterinário, Professor Adjunto, Escola de Veterinária da UFMG, Belo Horizonte, MG.

${ }^{3}$ Médico Veterinário, Professor, Doutor, FMVZ/USP, Pirassununga, SP.

${ }^{4}$ Médico Veterinário, Professor Associado, FMVZ, USP.

${ }^{5}$ Médico Veterinário, Professor, Doutor, Faculdade de Medicina Veterinária da UNISA, São Paulo, SP. 
Sudeste, utilizando-se eqüídeos em todos os estádios parasitários, caracterizados por picos populacionais definidos ao longo do ano (SERRA-FREIRE, 1982; SOUZA, 1990; OLIVEIRA, 1998; LABRUNA et al., 2002). Esse padrão deve-se, possivelmente, à influência de fatores climáticos na atividade dos diferentes estádios do carrapato nas pastagens (LABRUNA, 2000).

SOUZA (1990), OLIVEIRA (1998), OLIVEIRA et al. (2000) e LABRUNA et al. (2002) relataram que a dinâmica populacional do $\boldsymbol{A}$. cajennense na Região Sudeste caracteriza-se por predomínio de larvas nos meses de abril a julho, ninfas de julho a outubro e adultos de outubro a março. Apesar disso, observações em áreas severamente infestadas demonstram que, nos meses de outono e inverno, quando predominam as formas de larva e ninfa, praticamente não são realizados tratamentos químicos nos eqüinos, uma vez que, nestes estádios, os ácaros apresentam tamanho reduzido, ao ponto de uma infestação moderada não despertar a atenção do criador, pois mesmo ingurgitadas, as larvas e ninfas ficam sob o pêlo dos animais. Durante os meses de primavera e verão, quando o estádio adulto predomina, este chama muito a atenção do criador, dado ao grande volume que as fêmeas ingurgitadas atingem. Por esta razão, é nesta época que os banhos carrapaticidas predominam nas eqüideoculturas onde a infestação pelo A. cajennense é um problema. Mesmo assim, o sucesso do controle químico de $\boldsymbol{A}$. cajennense em eqüinos tem sido um constante desafio para o criadores (LABRUNA, 2000).

As formulações comerciais à base de piretróides são as únicas indicadas no comércio brasileiro para banhos carrapaticidas em equiídeos. Ensaios a campo e em laboratório demonstraram que a fase adulta de A. cajennense é naturalmente mais resistente às formulações comerciais desta base (PINHEIRO, 1987). Um aspecto importante é a curta duração das fases parasitárias de A. cajennense sobre os hospedeiros. Cada larva ou ninfa alimenta-se por apenas quatro a cinco dias em média, e as fêmeas por apenas sete a 10 dias (LOPES et al., 1998; PINTER et al., 2002). O banho carrapaticida efetivo, por outro lado, deve disponibilizar, no mínimo, quatro a cinco litros de calda carrapaticida por eqüino adulto, seguindo-se as recomendações dos fabricantes. No entanto, observações a campo têm demonstrado que grande parte dos criadores banham os eqüinos com volumes de calda carrapaticida bem inferiores aos recomendados (LABRUNA, 2000).

Baseando-se nestes fatores, LEITE et al. (1997) propuseram, para eqüinos, um programa de controle químico do A. cajennense por meio de banhos carrapaticidas semanais durante os meses de abril a outubro, quando predominam as formas de larva e ninfa deste carrapato. $\mathrm{O}$ presente trabalho objetivou quantificar o efeito desta estratégia de controle sobre uma população de $\boldsymbol{A}$. cajennense em pastagens ocupadas por eqüinos no Campus da Universidade de São Paulo, Município de Pirassununga, Estado de São Paulo.

\section{MATERIAL E MÉTODOS}

A pastagem de suporte, durante o monitoramento dos eqüinos, que originalmente apresentava aproximadamente oito ha em setembro de 1997, foi subdividida em duas, por uma cerca de arame farpado (designadas como pastagens 1 e 2), que apresentavam composição vegetal similar. As gramíneas predominantes eram grama-batatais (Paspalum notatum) e capim-brachiaria (Brachiaria decumbens). No entanto, abundavam outros tipos de vegetação: guanxuma (Sida spp), arranha-gato (Acacia bonariensis), fedegoso (Senna occidentalis), mentrasto (Ageratum conyzoides), e várias árvores de porte grande que proporcionavam largas áreas de sombreamento. A área total era explorada por eqüinos desde 1997 (10 a 20 eqüinos), sem que houvesse qualquer tipo de intervenção mecânica. Os eqüinos presentes nas pastagens 1 e 2 sempre se apresentaram naturalmente infestados por A. cajennense, sendo que tratamentos carrapaticidas foram efetuados esporadicamente apenas nos eqüinos da pastagem 2, a intervalos variados. O presente trabalho iniciou-se em dezembro de 1999, quando cada uma das pastagens foi mantida com cinco éguas adultas, sem raça definida, até o final do experimento, em fevereiro de 2001, perfazendo uma densidade de 1,25 éguas/ha. No caso da pastagem 1 , observações adicionais foram realizadas até fevereiro de 2002.

Observações anteriores sobre a dinâmica populacional das fases parasitárias de A. cajennense sobre eqüinos na pastagem 1 indicaram que os maiores picos de carrapatos adultos ocorreram nos meses de janeiro e fevereiro de cada ano avaliado (LABRUNA et al., 2002). Por esta razão, para o presente estudo, as cargas parasitárias de adultos de A. cajennense foram inicialmente avaliadas em eqüinos, nas duas pastagens, durante os meses de janeiro e fevereiro de 2000. Posteriormente, os eqüinos da pastagem 1 foram tratados semanalmente, sempre às segundas-feiras, da primeira semana de abril à última semana de outubro de 2000, com o objetivo de atingir diretamente as fases de larva e ninfa de A. cajennense sobre os animais. Os 
eqüinos da pastagem 2 foram tratados semanalmente apenas da primeira semana de abril à última semana de julho de 2000, com o objetivo de atingir diretamente a fase de larva de $\boldsymbol{A}$. cajennense sobre os eqüinos (LABRUNA et al., 2002).

Todos os banhos carrapaticidas foram realizados com carrapaticida comercial à base do piretróide alfametrina ${ }^{\text {a }}$. Utilizou-se uma bomba costal manual com 20 litros de carga, contendo a emulsão carrapaticida na concentração de $0,01 \%$ de princípio ativo. Durante os tratamentos, os animais eram contidos e banhados individualmente com um mínimo de quatro litros de emulsão. Os banhos carrapaticidas foram executados de forma homogênea, segundo normas técnicas, sem omitir o interior das orelhas e o divertículo nasal dos eqüinos. As crinas das éguas foram sempre mantidas aparadas para facilitar a aplicação do carrapaticida nesta área do corpo.

Em janeiro e fevereiro de 2000 (antes dos tratamentos), foram realizadas três contagens de carrapatos adultos de A. cajennense, intervaladas de 21 dias, no lado esquerdo de cada eqüino. $\mathrm{O}$ número total de carrapatos foi multiplicado por dois, para estimativa do número total de carrapatos adultos por animal (OLIVEIRA, 1998). Em janeiro e fevereiro de 2001 (após os tratamentos), três novas contagens de carrapatos adultos, intervaladas de 21 dias, foram realizadas com o objetivo de avaliar o impacto dos banhos carrapaticidas entre duas gerações consecutivas de adultos de A. cajennense nas pastagens. Os animais mantidos na pastagem 1 permaneceram sem qualquer tratamento carrapaticida até janeiro/fevereiro de 2002, quando três novas contagens de carrapatos foram realizadas, para quantificar a carga da segunda geração de adultos, pós tratamentos. Para uma melhor avaliação do impacto dos banhos carrapaticidas na população de carrapatos da pastagem 1, os valores de três contagens de carrapatos efetuadas nos eqüinos em janeiro/fevereiro de 1999, obtidos no estudo de dinâmica populacional (LABRUNA et al., 2002), foram considerados.

Os valores de contagens individuais nos eqüinos obtidos antes e após os tratamentos carrapaticidas foram comparados pelo teste não paramétrico de Mann-Whitney. As proporções de carrapatos adultos machos e fêmeas presentes nos equiinos, antes e após os tratamentos carrapaticidas, foram comparadas através do teste de Qui-quadrado $\left(\chi^{2}\right)$.

\section{RESULTADOS}

As médias das três contagens de carrapatos nos eqüinos em janeiro/fevereiro de 2000, foram de 763,6 e 560,7 carrapatos adultos por eqüino das pastagens 1 e 2, respectivamente (Tabela 1 e 2). No ano anterior (janeiro/fevereiro de 1999), a média para os eqüinos da pastagem 1 foi de 724,4 carrapatos. Os banhos carrapaticidas ocasionaram uma diminuição significativa na população de carrapatos adultos para o período de janeiro/fevereiro de 2001 de ambas pastagens $(\mathrm{P}<0,01)$. No entanto, esta redução foi nitidamente mais pronunciada nos eqüinos da pastagem 1 (média de 78,3 carrapatos por eqüino) do que da pastagem 2 (média de 232,0 carrapatos por eqüino). Diante destes valores, a redução da carga média de carrapatos adultos entre as contagens de 2000 e 2001 foi de $89,7 \%$ para a pastagem 1 e $58,6 \%$ para a pastagem 2 . Nas contagens de 2002, quando os eqüinos da pastagem 1 passaram o ano de 2001 sem qualquer tratamento carrapaticida, o valor médio de carrapatos por eqüino foi de 140,0. Embora este valor ainda esteja significativamente inferior aos valores das contagens pré-tratamentos carrapaticidas $(\mathrm{P}<0,01)$, ele foi significativamente superior ao valor médio de janeiro/fevereiro de 2001 ( $\mathrm{P}<0,01)$.

Durante o experimento, as infestações por adultos de A. cajennense nas éguas foram caracterizadas por um número maior de machos em

Tabela 1 - Quantificação de carrapatos adultos Amblyomma cajennense, através de três contagens realizadas de 21/21 dias em cada ano, em eqüinos mantidos na pastagem 1, no Município de Pirassununga, SP.

\begin{tabular}{|c|c|c|c|c|c|c|c|c|c|c|c|c|}
\hline & \multicolumn{3}{|c|}{ Jan/Fev de 1999} & \multicolumn{3}{|c|}{ Jan/Fev de 2000} & \multicolumn{3}{|c|}{ Jan/Fev de 2001} & \multicolumn{3}{|c|}{ Jan/Fev de 2002} \\
\hline & Média & $\mathrm{DP}^{1}$ & Amplitude & média & $\mathrm{DP}^{1}$ & Amplitude & média & $\mathrm{DP}^{1}$ & Amplitude & média & $\mathrm{DP}^{1}$ & Amplitude \\
\hline Contagem 1 & 395,0 & 119,2 & $272-572$ & 689,2 & 294,7 & $300-989$ & 64,4 & 49,5 & $28-148$ & 116,8 & 50,8 & $58-164$ \\
\hline Contagem 2 & 799,2 & 248,5 & $448-1030$ & 764,2 & 236,0 & $450-1000$ & 103,8 & 87,1 & $25-238$ & 182,0 & 127,1 & $78-350$ \\
\hline Contagem 3 & 978,8 & 298,4 & $598-1320$ & 837,6 & 294,4 & $468-1206$ & 66,8 & 58,3 & $22-167$ & 121,0 & 74,6 & $56-235$ \\
\hline MÉDIA* & $724,4^{\mathrm{a}}$ & 333,1 & $272-1320$ & $763,6^{\mathrm{a}}$ & 263,5 & $300-1206$ & $78,3^{\mathrm{b}}$ & 64,7 & $22-238$ & $140,0^{\mathrm{c}}$ & 88,8 & $56-350$ \\
\hline
\end{tabular}

a desvio padrão

* letras diferentes na mesma linha significam diferença estatisticamente significante pelo teste não paramétrico de Mann Whitney ao nível de $5 \%$ de significância 
relação ao de fêmeas, em todas as contagens realizadas. Nas contagens de janeiro/fevereiro de 1999 e 2000 nos animais da pastagem 1 , o percentual de fêmeas adultas contadas nas éguas correspondeu a 40,1 e $46 \%$ do total de carrapatos adultos, respectivamente. $\mathrm{Na}$ pastagem 2, foi de $42 \%$ em janeiro/fevereiro de 2000. Em janeiro/fevereiro de 2001 (após os banhos carrapaticidas), o número de fêmeas contadas nas éguas das pastagens 1 e 2 correspondeu a 20,4 e 31,4\% do total de carrapatos contados, respectivamente. Estes valores demonstraram que, pelo menos, na pastagem 1, a proporção de fêmeas em relação aos machos foi significativamente reduzida após os banhos carrapaticidas $(\mathrm{P}<0,05)$, ao passo que na pastagem 2 , a redução desta proporção esteve próxima de ser significante $(\mathrm{P}=0,052)$. Em janeiro/fevereiro de 2000, a média do número de fêmeas contadas nas éguas da pastagem 1 foi de 351,2 ao passo que, em janeiro/ fevereiro de 2001, observou-se uma média de 16,0 fêmeas por animal. Estes valores demonstram que os banhos carrapaticidas ocasionaram uma redução de $95,4 \%$ da população de fêmeas adultas sobre os eqüinos daquela pastagem. Na pastagem 2 , a redução do número de fêmeas adultas neste mesmo período foi de $69,0 \%$. Nas contagens de 2002 (segunda geração de adultos pós tratamentos), o número de fêmeas contadas nos eqüinos da pastagem 1 correspondeu a $37 \%$ do total de carrapatos adultos. Esta proporção de fêmeas observada em 2002 foi significativamente superior $(\mathrm{P}<0,05)$ à observada em 2001 , porém foi estatisticamente semelhante $(\mathrm{P}>0,05)$ daquelas observadas antes dos tratamentos químicos, nas contagens de 1999 e 2000.

\section{DISCUSSÃO}

Os resultados apresentados comprovam a eficácia da proposta de controle estratégico de $\boldsymbol{A}$. cajennense sugerida por LEITE et al. (1997). Os eqüinos da pastagem 1 (tratamentos de abril a outubro/ 2000) estavam sendo mantidos, sem qualquer tipo de tratamento químico, desde 1997, o que fez com que, no início do experimento, em janeiro de 2000, a pastagem 1 se encontrasse com alta carga de carrapatos. Mesmo assim, os banhos carrapaticidas efetuados semanalmente, de abril a outubro, reduziram em quase $90 \%$ a carga de carrapatos adultos entre duas gerações de A. cajennense na pastagem 1. É interessante destacar que os efeitos dos tratamentos de 2000 foram evidenciados por, pelo menos, mais uma geração de carrapatos, uma vez que as contagens de 2002 (segunda geração de adultos pós tratamento) ainda se mantiveram acentuadamente inferiores aos valores pré-tratamentos.

Em relação à pastagem 2 (tratamentos de abril a julho/2000), embora a carga de carrapatos antes do tratamento fosse inferior à da pastagem 1 , o efeito dos banhos carrapaticidas de abril a julho teve um impacto menor na população de $\boldsymbol{A}$. cajennense, com redução de apenas cerca de $60 \%$. Estes resultados demonstram que, no primeiro ano de implantação de um programa de controle estratégico de $\boldsymbol{A}$. cajennense, em áreas altamente infestadas, os banhos carrapaticidas devem impreterivelmente abranger todo o período de predomínio de larvas e ninfas (abril a outubro). Possivelmente, após um ano de controle abrangendo tais fases, quando a carga de carrapatos adultos na pastagem estiver significativamente reduzida, como aqui demonstrado, os tratamentos carrapaticidas poderiam ser restringidos à época de predomínio das larvas (abril a julho), o que tornaria o programa menos árduo, mais prático, com menores custos de mão-de-obra e carrapaticidas, e menos exposição dos animais, do homem e do meio ambiente a produtos químicos.

Nas condições naturais, o crescimento da população de carrapatos está diretamente relacionado ao número de fêmeas ingurgitadas que se desprendem

Tabela 2 - Quantificação de carrapatos adultos Amblyomma cajennense, através de três contagens realizadas de 21/21 dias em cada ano, em eqüinos mantidos na pastagem 2, no Município de Pirassununga, SP.

\begin{tabular}{|c|c|c|c|c|c|c|}
\hline & \multicolumn{3}{|c|}{$\mathrm{Jan} / \mathrm{Fev}$ de 2000} & \multicolumn{3}{|c|}{ Jan/Fev de 2001} \\
\hline & Média & $\mathrm{DP}^{1}$ & Amplitude & Média & $\mathrm{DP}^{1}$ & Amplitude \\
\hline Contagem 1 & 477,0 & 158,1 & $303-704$ & 207,0 & 82,8 & $122-300$ \\
\hline Contagem 2 & 557,6 & 126,0 & $416-668$ & 245,2 & 103,5 & $134-366$ \\
\hline Contagem 3 & 647,7 & 196,5 & $504-987$ & 244,0 & 100,1 & $141-370$ \\
\hline MÉDIA* & $560,7^{\mathrm{a}}$ & 167,1 & $303-987$ & $232,0^{\mathrm{b}}$ & 90,1 & $122-370$ \\
\hline
\end{tabular}

${ }^{1}$ desvio padrão

* letras diferentes na mesma linha significa diferença estatisticamente significante pelo teste não paramétrico de Mann Whitney ao nível de $5 \%$ de significância 
do hospedeiro, entre duas gerações. No presente trabalho, foi observado que, pelo menos, na pastagem 1 , o percentual de redução do número de fêmeas adultas $(95,4 \%)$ parasitando os eqüinos, após os banhos carrapaticidas, foi significativamente maior do que a da população total de adultos $(89,7 \%)$. Essa diferença está possivelmente relacionada com a concentração de feromônios produzidos pelos carrapatos machos sobre o hospedeiro, como atrativo para as fêmeas infestantes no ambiente. Através de observações a campo e em laboratório, LABRUNA et al. (2002) e PINTER et al. (2002) sugeriram que quanto maior a quantidade de machos de $\boldsymbol{A}$. cajennense sobre os eqüinos, maior a concentração de feromônios secretados por estes. Em conseqüência, os eqüinos com alta carga de machos tornam-se mais atrativos para fêmeas do que aqueles com menores quantidades de machos. Como os banhos carrapaticidas ocasionaram uma redução da carga de carrapatos adultos nos eqüinos, estes tornaram-se menos atrativos para as fêmeas infestantes do ambiente, ocasionando uma redução ainda maior da quantidade de fêmeas de $\boldsymbol{A}$. cajennense parasitando os animais. Por outro lado, as contagens de 2002, que foram significativamente superiores às de 2001, a proporção de fêmeas em relação a machos foi superior à de 2001, porém semelhante às observadas nas contagens antes dos tratamentos carrapaticidas. Isto possivelmente se deveu ao aumento significante da população de adultos nesta pastagem.

A concentração do carrapaticida pitretróide utilizada nos banhos obedeceu à recomendação do fabricante, para o carrapato A. cajennense. Embora esta concentração fosse duas vezes maior que a concentração usualmente recomendada para o carrapato de bovinos (Boophilus microplus), não foi observado qualquer tipo de reação adversa após os banhos carrapaticidas nas éguas.

Embora não tenha sido possível manter um grupo controle de eqüinos sem qualquer tratamento químico, paralelamente aos grupos de eqüinos das pastagens 1 e 2 , há evidências de que a redução da carga de carrapatos nas pastagens 1 e 2 foi realmente desencadeada pelos banhos carrapaticidas. LABRUNA et al. (2001) demonstraram que altas infestações por A. cajennense nos eqüinos estão fortemente dependentes da presença de pastos "sujos", os quais possivelmente proporcionam condições ideais de microclima para sobrevivência e desenvolvimento deste carrapato. A área experimental manteve-se semelhante antes e durante o experimento, não havendo efeito da cobertura vegetal na redução da carga de carrapatos. Além disso, as taxas de lotação dessas pastagens se mantiveram as mesmas que vinham sendo adotadas antes do experimento, sendo inclusive, mantidas as mesmas éguas com várias infestações prévias e constantes por A. cajennense. Isto também descarta algum efeito significativo devido à resistência imunológica que esses animais pudessem ter adquirido contra o A. cajennense. Por último, em consulta à estação meteorológica da Faculdade de Zootecnia e Engenharia de Alimentos da Universidade de São Paulo, localizada a aproximadamente $150 \mathrm{~m}$ da área experimental, comprovou-se que, em termos climáticos gerais, o ano de 2000 não foi considerado atípico aos anos anteriores, descartando uma influência significativa de efeitos climáticos sobre as formas de A. cajennense nas pastagens.

O presente trabalho demonstra que a população de adultos de $\boldsymbol{A}$. cajennense pode ser significativamente reduzida com tratamentos carrapaticidas sobre os estádios imaturos do carrapato. Como estes estádios predominam nos meses de menores índices pluviométricos em grande parte do Brasil (abril a outubro), a possibilidade de se repetir os banhos carrapaticidas, a cada sete dias, com satisfatória eficácia, é maior nesta época do ano. Neste trabalho, as chuvas inviabilizaram a realização dos banhos carrapaticidas em apenas uma das Segundasfeiras do período de tratamento, quando as chuvas estenderam-se por todo o decorrer do dia. Nesta única ocasião, os banhos foram transferidos para o dia seguinte, Terça-feira.

Mesmo que o controle de uma população de A. cajennense tenha sido obtido através de banhos estratégicos e de forma intensiva, deve-se salientar que, uma vez mantidas as condições de pastagens "sujas", favoráveis ao desenvolvimento deste carrapato no ambiente, a população de $\boldsymbol{A}$. cajennense pode nunca ser extinta da área. Neste caso, parte da população de carrapatos poderia se manter na área, alimentando-se em algumas espécies de animais silvestres que possibilitem o seu desenvolvimento, pois estes animais também freqüentam mais intensamente pastagens "sujas", ns quais a cobertura vegetal é mais densa.

\section{AGRADECIMENTOS}

Agradecemos aos funcionários Antônio Santa Roza, José Roberto Devitto e Valdir da Silva pelo suporte técnico durante o estudo. Ao Laboratórios Pfizer Ltda. Divisão de Saúde Animal, por disponibilizar a formulação comercial de carrapaticida utilizada neste trabalho. Este trabalho foi parcialmente financiado pela FAPESP (Processo n. 95/6938-0). 


\section{FONTES DEAQUISIÇÃO}

a Ultimate, Pfizer

\section{REFERÊNCIAS BIBLIOGRÁFICAS}

ARAGÃO, H. Notas sobre ixódidas brazileiros. Mem Inst Oswaldo Cruz, v.3, p.145-195, 1911.

ARAGÃO, H. Ixodidas brasileiros e de alguns paizes limitrophes. Mem Inst Oswaldo Cruz, v.31, p.759-843, 1936.

LABRUNA, M.B. Aspectos da biologia e epidemiologia dos carrapatos de eqüinos no Estado de São Paulo. 2000. 76f. Tese (Doutorado em Epidemiologia Experimental e Aplicada a Zoonoses) - Curso de Pós-graduação em Epidemiologia Experimental e Aplicada a Zoonoses, Universidade de São Paulo.

LABRUNA, M.B. et al. Risk factors to tick infestations and their occurrence on horses in the State of São Paulo, Brazil Vet Parasitol, v.97, p.1-14, 2001.

LABRUNA M.B. et al. Seasonal dynamics of ticks (Acari: Ixodidae) on horses in the state of São Paulo, Brazil. Vet Parasitol, v.105, p.65-77, 2002.

LEITE, R.C. et al. Alguns aspectos epidemiológicos das infestações por Amblyomma cajennense : uma proposta de controle estratégico. In: VERÍSSIMO, C.J.; AUGUSTO, C. Simpósio sobre Controle de Parasitos, 2.: controle de parasitos de eqüinos. Colina, SP : Instituto de Zootecnia, 1997. p.9-14

LOPES, C.M.L. et al. Host specificity of Amblyomma cajennense (Fabricius, 1787) (Acari: Ixodidae) with comments on the drop-off rhythm. Mem Inst Oswaldo
Cruz, v.93, p.347-351, 1998.

OLIVEIRA, P.R. Amblyomma cajennense (Fabricius, 1787) (Acari: Ixodidae): Avaliação de técnicas para o estudo de dinâmica populacional e biotecnologia. 1998. 97f. Tese (Doutorado em Ciência Animal) - Curso de Pós-graduação em Medicina Veterinária, Universidade Federal de Minas Gerais.

OLIVEIRA, P.R. et al. Population dynamics of the free living stages of Amblyomma cajennense (Fabricius, 1787) (Acari: Ixodidae) on pastures of Pedro Leopoldo, Minas Gerais State, Brazil. Vet Parasitol, v.92, p.295-301, 2000.

PINHEIRO, V.R.E. Avaliação do efeito carrapaticida de alguns piretróides sintéticos sobre o carrapato Amblyomma cajennense (Fabricius, 1787) (Acarina: Ixodidae). 1987. 126f. Dissertação (Mestrado em Parasitologia Animal) - Curso de Pós-graduação em Medicina Veterinária - Parasitologia Veterinária, Universidade Federal Rural do Rio de Janeiro.

PINTER, A.S.; LABRUNA, M.B.; FACCINI, J.L.H. Feeding period of males and females of Amblyomma cajennense (Acari: Ixodidae) under laboratory conditions, with special reference to sex ratio. Vet Parasitol, v.104, p.79-88, 2002.

SERRA-FREIRE, N.M.S. Epidemiologia de Amblyomma cajennense: ocorrência estacional e comportamento dos estádios não parasitários em pastagens do Estado do Rio de Janeiro. Arq Univ Fed Rur Rio de Janeiro, v.5, p.187-193, 1982.

SOUZA, A.P. Variação populacional dos principais ixodídeos parasitas de bovinos e eqüinos em diferentes condições de manejo, nos municípios de Paracambi e Itaguaí no estado do Rio de Janeiro. 1990. 81f. Tese (Doutorado em Parasitologia Animal) - Curso de Pós-graduação em Medicina Veterinária - Parasitologia Veterinária, Universidade Federal Rural do Rio de Janeiro. 